\title{
PERÍODO MÍNIMO DE COLHEITA PARA AVALIAÇÃo DE CULTIVARES DE CACAU EM LINHARES-ES ${ }^{1}$
}

\author{
Luiz Antônio dos Santos Dias², Carlos Alberto Spaggiari Souza ${ }^{3}$, Sebastião Geraldo Augusto ${ }^{4}$, Paulo Roberto \\ Siqueira ${ }^{5}$ e Manfred Willy Müller ${ }^{6}$
}

\begin{abstract}
RESUMO - Investigou-se o período mínimo de colheitas sucessivas necessário para avaliação do desempenho de cultivares de cacau. Os cultivares locais não-melhorados 'Maranhão', 'Pará' e 'Parazinho', juntamente com os cultivares melhorados 'ICS 1' polinização livre e híbrido comercial, foram avaliados em quadrado latino $5 \times 5$ com parcelas de 196 plantas, durante dez anos (1984-93). O número de frutos sadios por planta (NFSP) e o peso de sementes úmidas por hectare (PSUH) e por fruto (PSUF) foram os componentes de produção avaliados. A análise dos coeficientes de correlação entre produções anuais e acumuladas no decênio mostrou a necessidade da combinação dos primeiros seis e oito anos de colheitas sucessivas para NFSP e PSUH, respectivamente, para avaliação dos cultivares. A ampla variabilidade e o desempenho superior no pós-clímax (1989-93) e a tendência de os cultivares interagirem com os anos no pré-clímax indicaram que a avaliação deve ser conduzida em cacaueiros cultivados em Linhares somente após o oitavo ano de cultivo.
\end{abstract}

Palavras-chave: Híbrido e cultivares de cacaueiro, período mínimo de colheita, coeficiente de correlação e Theobroma cacao L.

\section{MINIMUM HARVEST PERIOD FOR EVALUATION OF CACAO CULTIVARS IN LINHARES, ES}

\begin{abstract}
The minimum harvest period for evaluating cacao cultivars was investigated. 'Maranhão', 'Pará', 'Parazinho' (non-improved local cultivars) and ehte open-pollinated 'ICS I' and the commercial hybrid mixture (improved cultivars) were evaluated in a 5 × 5 Latin square design containing 196-plant plots, during 10 years (1984-1993). The number of healthy fruits per plant (NHFP) and the wet seed weight per hectare (WSWH) and per fruit (WSWF) were the yield components evaluated. The combination of the first six and eight years of successive harvests for NHFP and WSWH, respectively, was shown to be necessary for cultivar evaluation, when correlation analyses between annual yields and accumulated yield in the decennium were performed. Extensive variability, superior performance in post-climax (1989-1993) and cultivar trends towards interaction with pre-climax age indicated that evaluation of Linhares cacao cultivars should be conducted only after the 8th year from planting.
\end{abstract}

Key words: Commercial cacao tree hybrid mixture and cultivars, minimum harvest period, correlation coefficient, and Theobroma cacao $L$.

1 Recebido para publicação em 13.11.2002.

Aceito para publicação em 9.9.2003.

2 Doutor em Genética e Melhoramento de Plantas, Pesquisador, Departamento de Biologia Geral, BIOAGRO, Universidade Federal de Viçosa - UFV, 37570-000 Viçosa-MG, <lasdias@ ufv.br>. ${ }^{3}$ Doutor em Fitotecnia, Pesquisador, Estação Experimental "Filogônio Peixoto", Centro de Pesquisas do Cacau, Caixa Postal 102, 29901-970 Linhares-ES, <carlosspaggiari@ bol.com.br>. ${ }^{4}$ Doutor em Engenharia Agrícola, Professor, Campus Universitário de Altamira, Universidade Federal do Pará, Caixa Postal 134, 68370-000 Altamira-PA, <saugusto@amazoncoop.com.br>. ${ }^{5}$ Mestre em Irrigação e Drenagem, Pesquisador e Chefe da Estação Experimental "Filogônio Peixoto", Centro de Pesquisas do Cacau, Caixa Postal 102, 29901-970 Linhares-ES, <esfip@escelsa.com.br>. ${ }^{6}$ Ph.D. em Fisiologia Vegetal, Pesquisador, Escritório de Representação de Salvador, CEPLAC, Av. Sete de Setembro, 2209, Corredor da Vitória, 40.080-002 Salvador-BA, <mwmuller@ufba.br>. 


\section{INTRODUÇÃO}

O cacaueiro é cultivado na região do baixo rio Doce, no Estado do Espírito Santo, desde 1917, a partir de sementes procedentes do Estado da Bahia. São cacaueiros do grupo racial Forasteiro Amazônico, representado pelos cultivares Comum, Pará e Maranhão. 'Pará' e 'Maranhão' apresentam ainda formas ou tipos conhecidos como Parazinho, Maranhão Liso e Maranhão Rugoso, respectivamente. Soria (1963) considera que os cacaueiros classificados como pertencentes ao cultivar Comum sejam, na realidade, uma mistura que inclui o 'Pará', o 'Maranhão' e tipos intermediários, devido a hibridações naturais. A expansão do cultivo nas décadas seguintes exigiu material genético de elevado potencial produtivo. Optou-se, então, pela síntese de híbridos biclonais, envolvendo seleções clonais locais (SIC, SIAL e EEG) e seleções clonais introduzidas de outros países produtores, como SCA, UF, IMC, DR, dentre outras. A partir de 1966, sementes de uma mistura de híbridos produzidas na Bahia foram distribuídas aos cacauicultores daquele Estado e do norte do Espírito Santo (Vello et al., 1969).

Em 1969, a Comissão Executiva do Plano da Lavoura Cacaueira (CEPLAC), órgão incumbido da condução da política nacional de cacau, criou, através do seu Centro de Pesquisas do Cacau (CEPEC), a Estação Experimental "Filogônio Peixoto" (ESFIP), em Linhares, Espírito Santo. A ESFIP adotou o programa de melhoramento genético delineado para as condições do sul da Bahia, até que informações locais fossem geradas. Esse programa enfatizou a produção de híbridos de cacaueiros de maior capacidade produtiva, resistentes a doenças de importância local e dotados de sementes de elevado peso, visando maior rendimento industrial (Vello et al., 1969; 1972; Smith et al., 1990). Os esforços da pesquisa e da assistência técnica local proporcionaram, no período de 1955 a 1985, um aumento de 59\% na produtividade de amêndoas secas, para uma taxa de expansão de área cultivada inferior a 10\% (Smith et al., 1990). Esses resultados mostram que a introdução ou a adaptação de tecnologias concorreu para um significativo incremento da produção de cacau do Estado. Regionalmente, os cultivares melhorados apresentam desempenho produtivo superior ao dos cultivares tradicionais não-melhorados (Pereira et al., 1987; Dias et al., 1998, 1999a), aliado à maior estabilidade produtiva no tempo (Dias et al., 1998; 1999b).

A despeito do relativo êxito alcançado com o programa de melhoramento do cacaueiro, é necessário lembrar que a produção é um caráter muito variável, de natureza quantitativa, composta de alguns componentes e altamente influenciada pelo ambiente (Soria, 1978). Deste modo, o procedimento para avaliação e seleção de cacaueiros superiores em produção baseia-se em registros de colheitas quinzenais ou mensais sucessivas, durante dois ou mais anos (Pound, 1932, 1933; Cheesman \& Pound, 1934; Pinto et al., 1969). Entretanto, o período mínimo de tempo de colheitas sucessivas necessário para que a avaliação e a seleção de genótipos nas condições locais sejam praticadas eficientemente não é suficientemente bem conhecido. Para realçar a importância desse conhecimento basta lembrar que a experimentação de campo com espécies arbóreas como o cacaueiro é freqüentemente morosa, tediosa e onerosa (Dias \& Resende, 2001). Em geral, os experimentos com cacaueiros demandam tempo e área excessivos, representando elevados custos com implantação, manejo, colheita e mão-de-obra. Por estas razões, a simplicidade, a flexibilidade e a robustez do delineamento experimental a ser utilizado e a multiplicidade de objetivos da pesquisa são aspectos importantes que devem ser observados na experimentação com o cacaueiro (Dias \& Resende, 2001).

No presente trabalho, um ensaio comparativo envolvendo híbridos de cacaueiro recomendados para plantio no ano de 1982 e quatro outros cultivares (sendo três locais e um introduzido) foi avaliado durante dez anos (1984-93). Investigou-se o desempenho desses cultivares com base em três componentes de produção, em dois períodos arbitrários denominados pré-clímax (198488) e pós-climax (1989-93) produtivo. Em seguida, determinou-se o período mínimo de colheitas sucessivas necessário para proceder à avaliação e à seleção com eficiência.

\section{MATERIAL E MÉTODOS}

\subsection{Procedimentos Experimentais}

Cinco cultivares de cacau foram ensaiados na ESFIP. Esses cultivares e seus grupos raciais estão relacionados a seguir:

1) 'Maranhão' - Forasteiro do Alto Amazonas;

2) 'Pará' - Forasteiro do Baixo Amazonas;

3) 'Parazinho' - Forasteiro do Baixo Amazonas;

4) 'ICS 1' polinização livre - Trinitário, selecionado em Trinidad pelo Imperial College of Tropical Agriculture; $\mathrm{e}$ 
5) 'Híbrido comercial' - Mistura constituída de pelo menos cinco híbridos, envolvendo clones da Bahia (séries SIC e SIAL) e do Espírito Santo (série EEG), em cruzamento com clones introduzidos, como SCA, UF, IMC, DR, dentre outros.

Detalhes que envolvem a obtenção das sementes desses cultivares e a caracterização completa do local de estudo (clima, solo e localização) estão descritos em Dias et al. (1998; 1999a, b). O ensaio foi instalado em fevereiro de 1982, em quadrado latino 5 × 5, com parcelas de 196 cacaueiros, espaçados a cada $3 \mathrm{~m}$. O sombreamento temporário foi realizado com bananeiras, em espaçamento similar ao dos cacaueiros. O sombreamento definitivo foi realizado com boleira (Joannesia princeps Vell.) plantada em espaçamento $12 \times 12 \mathrm{~m}$. Todas as práticas de manejo preconizadas para o cultivo do cacaueiro foram realizadas no experimento, exceto a adubação. As colheitas foram iniciadas em abril de 1984 e realizadas mensalmente, durante 10 anos (1984-93). Entretanto, para efeito de análise, utilizou-se a produção dos anos agrícolas (abril/março). Os componentes de produção estudados foram o número de frutos sadios por planta (NFSP), o peso de sementes úmidas por hectare (PSUH), $\mathrm{em} \mathrm{kg} / \mathrm{ha}$, e o peso de sementes úmidas por fruto (PSUF), em g/fruto.

\subsection{Procedimentos Estatísticos}

As análises de variância foram processadas para dois períodos de tempo, arbitrariamente estabelecidos, denominados pré (1984-88) e pós-clímax (1989-93) produtivo. Foram analisados os três componentes de produção, utilizando o arranjo de parcelas subdivididas no tempo, alocando-se o quadrado latino nas parcelas e os anos nas subparcelas (Steel et al., 1997). A influência relativa das várias fontes de variação sobre a diversidade total dos dados foi medida pelo coeficiente de determinação $\left(\mathrm{R}^{2}\right)$, a partir das somas de quadrado (Vencovsky \& Barriga, 1992). Também foram estimados os coeficientes de correlação entre as médias de produções e médias de produções anuais acumuladas sucessivas com as médias de produções obtidas no decênio. Todas as análises foram efetuadas no progama SAS (SAS Institute Inc, 1989).

\section{RESULTADOS E DISCUSSÃO}

A fisiologia do cacaueiro apresenta períodos com relativa definição. Do plantio ao início da produção econômica tem-se o período juvenil, cuja duração varia entre três e cinco anos. Por sua vez, o período produtivo, que sucede ao juvenil, pode ser dividido em período pré e pós-clímax. O clímax traduz a maximização do potencial de produção e, de modo geral, ocorre entre o oitavo e o décimo ano de plantio (Reyes \& Perez, 1971). Na avaliação do desempenho dos cultivares ao longo do período de estudo (1984-93) foi estabelecida uma divisão arbitrária, contemplando dois períodos: 1984-88 e 198993. O primeiro período, compreendido entre o terceiro e o sétimo ano de plantio, permitiu investigar o desempenho no pré-clímax, enquanto no segundo $\left(8^{\circ}\right.$ ao $12^{\circ}$ ano de plantio) investigou-se o desempenho no pós-clímax.

As médias e amplitudes obtidas pareceram confirmar a existência de dois períodos produtivos distintos (Quadro 1). No geral, os cultivares exibiram médias e amplitudes menores no período pré-clímax que no pósclímax. Essa distinção foi acentuada para NFSP e PSUH. É bem provável que as condições adversas de

Quadro 1 - Valores de médias e de amplitude dos componentes de produção avaliados em cinco cultivares de cacau, em três períodos

Table 1 - Mean and range values of yield components in five cacao cultivars over three periods of time

\begin{tabular}{|c|c|c|c|c|c|c|}
\hline \multirow{3}{*}{ Caráter } & \multicolumn{6}{|c|}{ Ano } \\
\hline & \multicolumn{2}{|c|}{$1984-88$} & \multicolumn{2}{|c|}{$1989-93$} & \multicolumn{2}{|c|}{ 1984-93 } \\
\hline & Média & Amplitude & Média & Amplitude & Média & Amplitude \\
\hline $\mathrm{NFSP}^{1 /}$ & 4,3 & $0,1-23,2$ & 9,1 & $0,3-41,3$ & 6,7 & $0,1-41,3$ \\
\hline $\mathrm{PSUH}^{2}{ }^{2 /}$ & 356,5 & $15,6-1812,7$ & 922,3 & $21,1-3712,7$ & 639,4 & $15,6-3712,7$ \\
\hline PSUF $^{3 /}$ & 77,8 & $44,3-128,3$ & 81,2 & $41,8-121,9$ & 79,5 & $41,8-128,3$ \\
\hline
\end{tabular}

${ }^{1 /}$ número de frutos sadios por planta, ${ }^{2 /}$ peso de sementes úmidas por hectare, em $\mathrm{kg} / \mathrm{ha}, \mathrm{e}{ }^{3 /}$ peso de sementes úmidas por fruto, em g/fruto. 
(Pereira et al., 1987; Mariano et al., 1988). Com base nos valores de $\mathrm{R}^{2}$, que mede a contribuição relativa das fontes de variação para a dispersão dos dados, verificouse que Ano foi a principal fonte de variação, para ambos os períodos. Logo, a série de anos contemplada pelo estudo foi muito ampla. Convém ressaltar que a análise tradicional de variância e a análise de medidas repetidas, ambas com o quadrado latino em parcelas subdivididas no tempo, foram processadas para todo o decênio (198493), para PSUH (Dias et al., 2001). Em razão da significância do teste de esfericidade, os testes multivariados e os univariados ajustados foram recomendados, muito embora ambos tenham revelado significância das probabilidades associadas aos testes F para os efeitos de Ano e Ano x Cultivar.

Dias et al. (1998; 1999a), ao investigarem o desempenho desses cultivares no mesmo ensaio, relataram que as médias das produções exibiram tendência de aumento com o avanço na idade dos cacaueiros e grande variabilidade entre os anos, sobretudo para NFSP e PSUH, ao longo de todo o decênio. Ainda segundo aqueles autores, as condições climáticas locais adversas, particularmente o déficit hídrico, reduziram as produções de todos os cultivares. O híbrido comercial e o ICS 1 polinização livre foram superiores aos cultivares locais em PSUH e PSUF, respectivamente, sobretudo em anos com distribuição regular de chuva. Essa superioridade deveuse ao incremento no PSUF, gerando maior produção por área. Da mesma forma, estes dois cultivares se apresentaram como os mais estáveis e adaptados temporalmente, com relação ao PSUH (Dias et al., 1998; 1999b). Outro estudo (Carvalho, 1999) corroborou a assertiva anterior de que os cacaueiros híbridos de melhor desempenho médio são também os mais estáveis. Esses resultados indicam que o melhoramento praticado para o aumento de produtividade contribuiu também para maior estabilidade dos cultivares no tempo e reforçam a importância de se empregar cultivares melhorados no plantio. Em se tratando de cultura perene, os benefícios advindos do uso de materiais melhorados são duradouros e têm custo zero, já que as sementes desses genótipos são distribuídas gratuitamente aos cacauicultores pelo CEPEC.

O período mínimo de colheitas sucessivas para avaliação do potencial de produção dos cultivares foi investigado, estimando-se as correlações entre as médias das produções anuais e combinações de médias das produções sucessivas e as médias das produções obtidas no decênio (Quadro 3). Para o componente PSUF, a produção de um simples ano foi suficiente para avaliar o potencial produtivo dos cultivares $(\mathrm{r} \geq 0,90)$. Porém, valores altos de correlação foram obtidos somente a partir do sexto ano de produções sucessivas para NFSP e a partir do oitavo ano para PSUP, o que mostra a inconsistência da expressão desses caracteres nos vários anos. Para Atanda (1972), um período que compreende os dois até os cinco primeiros anos de produção acumulada de frutos se mostrou adequado para avaliar o potencial produtivo de cultivares de cacaueiros ensaiados na Nigéria. Dias \& Kageyama (1998), estudando cacaueiros híbridos ensaiados no sul da Bahia, obtiveram resultados semelhantes aos de Atanda (1972), no caso dois anos. Demonstrou-se com tais resultados que a avaliação e a seleção de cultivares de cacaueiro sob as condições locais devem ser conduzidas sobre vários anos de colheitas sucessivas, para serem eficientes. Esse fato é bem conhecido em cacau, sendo recomendável a combinação da produção de anos sucessivos para minimizar os efeitos anuais.

Quadro 3 - Coeficientes de correlação entre as médias de produções anuais (A) e médias de produções anuais acumuladas sucessivas (B) e as médias das produções do decênio (1984-93), referentes a cinco cultivares de cacau avaliados por três componentes de produção

Table 3 - Correlation coefficients between annual average yields (A) and accumulated average yields (B) from 1984 to 1993, in five cacao cultivars evaluated for three yield components

\begin{tabular}{|c|c|c|c|c|c|c|}
\hline \multirow{2}{*}{ Ano } & \multicolumn{2}{|c|}{ NFSP $^{1 /}$} & \multicolumn{2}{c|}{ PSUH $^{2 /}$} & \multicolumn{2}{c|}{ PSUF $^{3 /}$} \\
\cline { 2 - 7 } & A & B & A & B & A & B \\
\hline 1984 & 0,79 & 0,79 & 0,57 & 0,57 & 0,98 & 0,98 \\
1985 & 0,53 & 0,62 & 0,21 & 0,35 & 0,99 & 0,55 \\
1986 & 0,71 & 0,66 & 0,47 & 0,39 & 0,96 & 0,99 \\
1987 & 0,78 & 0,67 & 0,34 & 0,39 & 0,98 & 0,99 \\
1988 & 0,71 & 0,68 & 0,31 & 0,38 & 0,97 & 0,99 \\
1989 & 0,98 & 0,74 & 0,83 & 0,43 & 0,97 & 0,99 \\
1990 & 0,77 & 0,75 & 0,48 & 0,44 & 0,90 & 0,99 \\
1991 & 0,33 & 0,93 & 0,83 & 0,92 & 0,99 & 0,99 \\
1992 & 0,51 & 0,99 & 0,87 & 0,99 & 0,99 & 0,99 \\
1993 & 0,41 & 1,00 & 0,89 & 1,00 & 0,99 & 1,00 \\
\hline
\end{tabular}

1/ número de frutos sadios por planta, ${ }^{\stackrel{2}{ }}$ peso de sementes úmidas por hectare, em $\mathrm{kg} / \mathrm{ha}, \mathrm{e}^{\underline{3} /}$ peso de sementes úmidas por fruto, em g/fruto.

\section{CONCLUSÃO}

A inconsistência na expressão dos caracteres no período de estudo, quantificada pela correlação entre

R. Árvore, Viçosa-MG, v.27, n.4, p.495-501, 2003 
médias das produções anuais e médias de produções acumuladas sucessivas e entre essas e as produções obtidas no decênio, exige a combinação de anos de colheitas sucessivas para maior eficiência no processo de avaliação e seleção de cultivares.

O estabelecimento arbitrário do clímax produtivo no oitavo ano de cultivo permitiu dividir o decênio em períodos pré e pós-clímax, cuja existência foi confirmada pela avaliação do desempenho dos cultivares. A ampla variabilidade e o desempenho superior no pós-clímax (1989-93), aliados à tendência de os cultivares interagirem com os anos no pré-clímax, reforçaram a tese de que a avaliação, a seleção e a estimação de parâmetros genéticos devem ser conduzidas em cacaueiros locais no pósclímax, ou seja, após o oitavo ano de cultivo.

\section{AGRADECIMENTO}

Ao $\mathrm{CNPq}$, pelo apoio às nossas linhas de pesquisa e pela bolsa de produtividade concedida ao primeiro autor. Ao Bernardo Conceição e ao Sebastião Trindade dos Reis, técnico agrícola e prático de campo da ESFIP, respectivamente, pela dedicação e pelo empenho no manejo e na colheita do experimento, durante os últimos 13 anos.

\section{REFERÊNCIAS BIBLIOGRÁFICAS}

ATANDA, O. A. Correlation studies in Theobroma cacao L. Turrialba, v. 22, n. 1, p. 81-89, 1972.

BARTLEY, B. G. D. Yield variation in the early productive years in trials with cacao (Theobroma cacao L.).

Euphytica, v. 19, n. 2, p. 199-206, 1970.

BARTLEY, B. G. D.; MONTEIRO, W. R.; CARLETTO, G. A. Comportamento dos clones introduzidos como progenitores de híbridos na Bahia. In: CONFERÊNCIA INTERNACIONAL DE INVESTIGACIÓN EN CACAO, 8., 1981, Cartagena. Actas... London: Cocoa Producers' Alliance, 1982. p. 703-712.

CARVALHO, C. G. P. Repetibilidade e seleção de híbridos de cacaueiro. 1999. 177 f. Tese (Doutorado em Genética e Melhoramento) - Universidade Federal de Viçosa, Viçosa, 1999.

CHEESMAN, E. E.; POUND, F. J. Further notes on criteria of selection in cacao. ICTA Annual Report on Cacao Research, v. 3, p. 21-24, 1934.

R. Árvore, Viçosa-MG, v.27, n.4, p.495-501, 2003
DIAS, L. A. S.; KAGEYAMA, P. Y. Repeatability and minimum harvest period of cacao (Theobroma cacao L.) in Southern Bahia. Euphytica, v. 102, n. 1, p. 29-35, 1998.

DIAS, L. A. S.; RESENDE, M. D. V. Experimentação no melhoramento. In: DIAS, L. A. S. Melhoramento genético do cacaueiro. Viçosa: L.A.S. DIAS/FUNAPE-UFG, 2001. p. $439-492$.

DIAS, L. A. S.; CRUZ, C. D.; CARNEIRO, P. C. S. Analysis of experiments with repeated measures. INGENIC Newsletter, v. 6, p. 29-31, 2001.

DIAS, L. A. S. et al. Performance and temporal stability analyses of cacao cultivars in Linhares, Brasil. Plantations, Recherche, Développement, v. 5, n. 5, p. 343-355, 1998.

DIAS, L. A. S. et al. Desempenho de cultivares de cacau com relação a alguns componentes de produção em Linhares, ES, Brasil. In: INTERNATIONAL COCOA RESEARCH CONFERENCE, 12., 1996, Salvador. Proceedings... Lagos: Cocoa Producers' Alliance, 1999a. p. $449-450$.

DIAS, L. A. S. et al. Estabilidade temporal de cultivares de cacau com relação à produção em Linhares, ES, Brasil. In: INTERNATIONAL COCOA RESEARCH CONFERENCE, 12., 1996, Salvador. Proceedings... Lagos: Cocoa Producers' Alliance, 1999b. p. 451-457.

MARIANO, A. H.; YAMADA, M. M.; PEREIRA, M. G. Comportamento de híbridos de cacau sob distintas condições de clima e solo. In: CONFERÊNCIA INTERNACIONAL DE INVESTIGACIÓN EN CACAO, 10., 1987, Santo Domingo. Actas... Lagos: Cocoa Producers'Alliance, 1988. p. 627-633.

PEREIRA, M. G.; CARLETTO, G. A.; DIAS, L. A. S. Avaliação de híbridos de cacaueiros nas condições de Linhares-ES. Ilhéus: CEPLAC/CEPEC, 1987. 40 p. (Boletim Técnico, 150).

PINTO, G. C. P. et al. Seleção de matrizes superiores nos cacauais da Bahia. In: CONFERÊNCIA INTERNACIONAL DE PESQUISAS EM CACAU, 2., 1967, Salvador e Itabuna. Memórias... Ilhéus: CEPLAC, 1969. p. $105-113$.

POUND, F. J. The principles of cocoa selection. Proceedings of the Agricultural Society of Trinidad and Tobago, v. 32, n. 4, p. 112-127, 1932.

POUND, F. J. Criteria and methods of selection in cacao. ICTA Annual Report on Cacao Research, v. 2, p. 27-29, 1933. 
REYES, H.; PEREZ, A. Resultados de un ensayo comparativo de progenies hibridas enter cacao amazonico por criollo, amazonico por trinitario y criollo por trinitario. In: INTERNATIONAL COCOA RESEARCH CONFERENCE, 3., 1969, Accra. Proceedings... Tafo: Cocoa Research Institute of Nigeria, 1971. p. 590-599.

SAS Institute Inc. SAS/STAT user's guide. Version 6. 4.ed., v. 2, Cary: 1989. 846 p.

SMITH, G. E. (Ed.). Cultivo do cacaueiro no Estado do Espírito Santo. Ilhéus: CEPLAC/CEPEC, 1990. 52 p.

SORIA, J. Observaciones sobre las variedades y cultivares de cacao en Bahia, Brasil. Cacao, v. 8, p. 1-6, 1963.

SORIA, J. The breeding of cacao (Theobroma cacao L.). Tropical Agriculture Research Series, v. 11, p. 161-168, 1978.
STEEL, R. G. D.; TORRIE, J. H.; DICKEY, D. A. Principles and procedures of statistics: a biometrical approach. 3.ed. New York: McGraw-Hill, 1997. 666 p.

VELLO, F.; GARCIA, J. R.; MAGALHÃES, W. S.

Produção e seleção de cacaueiros híbridos na Bahia.

Revista Theobroma, v. 2, n. 3, p. 15-35, 1972.

VELLO, F. et al. O programa de melhoramento genético do cacau na Bahia. In: CONFERÊNCIA INTERNACIONAL DE PESQUISAS EM CACAU, 2. 1967, Salvador e Itabuna. Memórias... Ilhéus: CEPLAC, 1969. p. 43-56.

VENCOVSKY, R.; BARRIGA, P. Genética biométrica no fitomelhoramento. Ribeirão Preto: Revista Brasileira de Genética, 1992. 496 p. 